\title{
Physical activity in healthy urban adults and its association with body composition parameters
}

\author{
Rudite Lagzdina* and Maija Rumaka \\ Rīga Stradiņš University, Department of Human Physiology and Biochemistry, Riga, Latvia
}

\begin{abstract}
An adequate amount of physical activity (PA) in adults improves cardio-respiratory fitness, metabolic health and helps maintain a normal body weight and composition. Here we examined sex- and occupationrelated PA patterns and their association with body composition parameters.

Healthy volunteers $(n=97)$ aged 21-49 years completed a selfadministered PA questionnaire and characterized their occupational duties depending on the volume of the physical work involved. A bio-impedance analyser was used to determine body composition. Men reported more metabolic equivalent of task (MET) minutes/week of vigorous PA than women $(p<0.05)$.

Participants with low occupational PA had less walking, moderate and total MET minutes/week, and more sitting time than those with moderate and vigorous occupational PA $(p<0.05)$, but their body composition parameters did not differ.

In males, negative correlations were found between body fat percentage, visceral fat range, segmental fat mass, and moderate MET minutes/week, whereas positive associations were observed between muscle mass in legs and vigorous MET minutes/week $(p<0.05)$. In females, negative correlations were found between visceral fat and vigorous MET minutes/week but positive - between lean body mass, trunk and leg muscle mass, and moderate PA $(p<0.05)$. These observed associations might be used to develop interventions to promote sex- and occupation-specific PA.
\end{abstract}

Key words: physical activity, body composition, healthy adults, occupational physical activity, IPAQ.

\section{Introduction}

An adequate amount of physical activity (PA) in adults improves their cardio-respiratory fitness and metabolic bone health and helps them maintain a normal body weight and composition. A randomized controlled study demonstrated that lifestyle changes including increased PA is the primary intervention for maintaining an appropriate body weight and reducing excess visceral and upper body fat, the presence of which has a strong correlation with metabolic syndrome [1, 2]. In addition to leisure time, domestic and transport-related activities, daily PA substantially depend on occupational PA (OPA). Many people in urban areas are employed in professions with negligible PA, which can be considered a potential risk factor for decreased total daily PA.

Changes in society such as mechanization and motorization have decreased the OPA, transportation PA, and home PA. A longitudinal study of a Finnish adult population revealed

\footnotetext{
* Corresponding author: Rudite.Lagzdina@rsu.lv
} 
a decreased OPA and increased prevalence of leisure-time PA over a 30-year period [3]. However, data regarding long term changes in leisure time PA are inconsistent. Some population studies reported that leisure time PA remained at the same level, while others that it increased, but the results varied among geographical regions due to age and education level [4]. A systematic review published in 2017 identified evidence of a positive relationship between living in urban areas and greater sitting time and total sitting behaviour [5].

The present study evaluated the pattern of PA in a typical week to assess aspects related to OPA and body composition parameters among healthy adults involved in professional duties in an urban environment such as office work, health, recreation, education, and service. A cross-sectional study design was used to collect self-reported PA data and objective measurements of body parameters.

\section{Material and methods}

Participants were recruited using a chain-referral sampling technique. The study included adult volunteers of both sexes who met the following inclusion criteria: healthy, age 20-50 years, not using any medication during study participation, and engaged in typical occupational routines (e.g., not during annual holiday or leave). The study was approved by the Ethics Committee of Rīga Stradinš̌ University and informed consent was provided by each participant upon study entry. The PA of the participants was assessed using the International Physical Activity Questionnaire, short form (IPAQ-SF). Participants reported information about their PA in the previous 7 days and four domains of PA were analysed sitting, walking, and moderate and vigorous PA. In the further data processing, metabolic equivalent of task (MET) minutes for each activity were calculated. Self-reported PA data were handled according to the procedure described in the Guidelines for Data Processing and Analysis of the IPAQ [6]. In addition, participants were asked to characterize the type of their occupational duties depending on the volume of the physical work involved.

The body weight and height of each participant was measured to the nearest $0.01 \mathrm{~kg}$ and $1.0 \mathrm{~cm}$, respectively, and body mass index (BMI) was calculated. A Tanita MC-180 MA (Tanita Corp., Tokyo, Japan) multi-frequency bio-impedance analyser was used to determine body mass and body composition, including fat and muscle mass segmental distribution in each extremity and the trunk. To obtain more precise results, the measurements were taken in the morning after an overnight fast with limited PA before the test.

The statistical analysis was performed using SPSS Statistics v. 22.0 (IBM Corporation, USA) with significance set at $p<0.05$. The analysis included descriptive statistics for the entire sample stratified by sex and occupational groups in all cases. The results are presented as mean and standard deviation or median with interquartile range. Parameter differences between sexes and groups were assessed using an independent samples t-test in cases of Gaussian data distribution and the Mann-Whitney test in cases of non-Gaussian data distribution. Pearson chi-square and Spearman's tests were used to assess the relationship between variables.

\section{Results}

The study sample consisted of 97 participants and contained more women (55\%) than men. The anthropometric characteristics of the participants are presented in Table 1. Mean age, height, body mass, and BMI were higher among men $(p<0.05$, independent-samples t-test). According the answers about work-related PA, 64 (66\%) of the subjects had low OPA and $33(34 \%)$ had moderate-high OPA. There were no statistically significant differences in the mean values of the four mentioned anthropometric parameters among the OPA groups. 
Table 1. Descriptive characteristics of the study participants.

\begin{tabular}{|l|c|c|}
\hline & $\begin{array}{c}\text { Male }(\boldsymbol{n}=\mathbf{4 4}) \\
\text { mean } \pm \text { SD }\end{array}$ & $\begin{array}{c}\text { Female }(\boldsymbol{n}=\mathbf{5 3}) \\
\text { mean } \pm \mathbf{S D}\end{array}$ \\
\hline Age $($ years $)$ & $31.4 \pm 5.4$ & $29.0 \pm 5.7$ \\
\hline Height $(\mathrm{cm})$ & $183.5 \pm 6.7$ & $169.9 \pm 5.9$ \\
\hline Body mass $(\mathrm{kg})$ & $81.6 \pm 10.6$ & $63.7 \pm 7.1$ \\
\hline BMI $\left(\mathrm{kg} / \mathrm{m}^{2}\right)$ & $24.2 \pm 2.7$ & $22.1 \pm 2.6$ \\
\hline
\end{tabular}

Table 2. Characteristics of physical activity assessed by the IPAQ-SF.

\begin{tabular}{|l|c|c|c|}
\hline Parameter & $\begin{array}{c}\text { Male } \\
\text { median [IQR] }\end{array}$ & $\begin{array}{c}\text { Female } \\
\text { median [IQR] }\end{array}$ & $\boldsymbol{p}$ value* \\
\hline Sitting in working day (min/day) & $390[240-480]$ & $360[240-495]$ & $\mathrm{ns}$ \\
\hline $\begin{array}{l}\text { Sitting in day off } \\
\text { (min/day) }\end{array}$ & $300[191-360]$ & $300[180-360]$ & $\mathrm{ns}$ \\
\hline $\begin{array}{l}\text { Walking } \\
\text { (MET-min/week) }\end{array}$ & $660[282-1386]$ & $907[479-2376]$ & $\mathrm{ns}$ \\
\hline $\begin{array}{l}\text { Moderate PA } \\
\text { (MET-min/week) }\end{array}$ & $480[205-1110]$ & $540[260-960]$ & $\mathrm{ns}$ \\
\hline $\begin{array}{l}\text { Vigorous PA } \\
\text { (MET-min/week) }\end{array}$ & $840[0-1890]$ & $240[0-1440]$ & 0.025 \\
\hline $\begin{array}{l}\text { Total PA } \\
\text { (MET-min/week) }\end{array}$ & $2625[1541-4412]$ & $2772[1541-4166]$ & $\mathrm{ns}$ \\
\hline
\end{tabular}

${ }^{*} p$ values show gender differences, comparisons performed by Mann-Whitney $\mathrm{U}$ test. $\mathrm{ns}-p>0.05$.

The IPAQ-SF results are summarized in Table 2. The reported time of PA in each domain was characterized by MET minutes spent on them in 1 week. Walking MET minutes were computed as reported walking minutes in 1 week multiplied by the intensity coefficient 3.3. For moderate and vigorous MET minutes, coefficients 4.0 and 8.0, respectively, were used. The total number of MET minutes/week was defined as the sum of walking, moderate, and vigorous MET minutes.

The summary of PA expressed as MET minutes/week showed that men had significantly more MET minutes/week of vigorous PA than women, whereas no sex-based differences were observed in the sitting and moderate PA domains. The median values of walking and moderate MET minutes/week were higher in women, but the difference was not statistically significant. Walking and moderate PA in women and vigorous PA in men were the activities that also contributed the most to the total PA MET minutes/week.

As proposed by the IPAQ data processing and evaluation guidelines, PA results were evaluated as three-level categorical scores [6]. An equal number of participants (45.4\%) met the criteria to be qualified as moderate or highly physically active, while $9.2 \%$ of participants were low active. Since there was no association between sex and occupational group (Pearson chi-square $\chi=1.634, p=0.201$ ), separate results of men and women in each occupational group were not analysed.

Analysis of the distribution of participants by OPA level between IPAQ PA categories revealed that, in the low PA category, all participants had a low OPA; in the moderate PA category, $36 \%$ had a moderate to high OPA; and in high PA category, $68 \%$ had a moderate to high OPA. The Pearson chi-square test results showed a statistically significant association between OPA group and PA score $(\chi=13.67, p=0.001)$. The mean reported sitting time in working days (minutes per day) was significantly higher in subjects in the low OPA group 

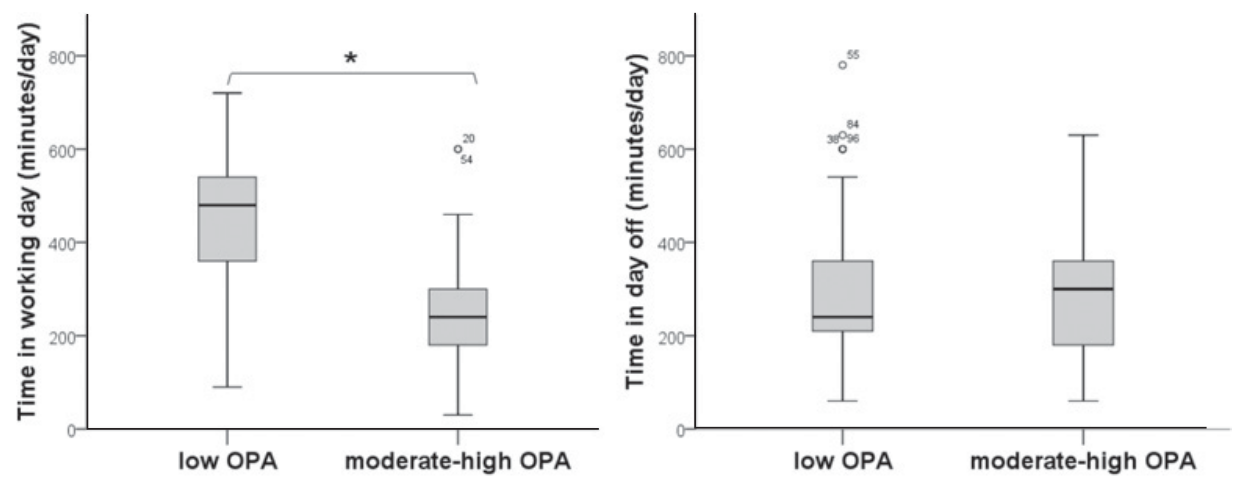

Figure 1. Sitting time by occupational physical activity (OPA) group in working day and in day off. ${ }^{*} p<0.05$, Mann-Whitney $\mathrm{U}$ test.

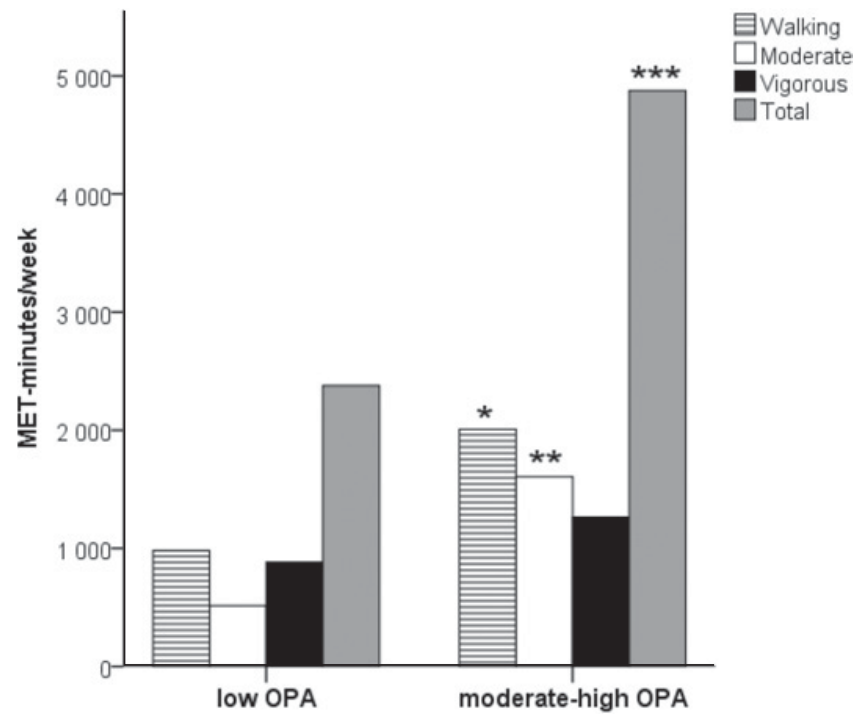

Figure 2. Physical activity domains of the occupational physical activity (OPA) groups. ${ }^{*, *, * * *} p<0.05$ $v s$. low OPA group, Mann-Whitney U test.

than in the moderate to high OPA group, whereas sitting time duration on days off was similar (Fig. 1).

Analysis of MET minutes of the separate PA domains in the occupational groups (Fig. 2) revealed that the subjects in the moderate to high OPA group had significantly higher results of walking, moderate, and total MET minutes/week than those in the low OPA group.

The relationship between PA domain and body composition parameters was assessed using the Spearman correlation test (Table 3). Since there were no statistically significant differences in analysed body parameters between the OPA groups, the data were stratified by sex only.

In men, a moderate MET minutes/week was inversely correlated with body fat, visceral fat range, and segmental fat mass of the trunk, arms, and legs, whereas a positive relationship was observed between muscle mass in the legs and vigorous activity MET minutes/week. 
Table 3. Correlation coefficients (Spearman's rho) between MET-minutes in physical activity domains and body composition parameters.

\begin{tabular}{|l|c|c|c|c|}
\hline & \multicolumn{2}{|c|}{ Male } & \multicolumn{2}{c|}{ Female } \\
\cline { 2 - 5 } & $\begin{array}{c}\text { Moderate } \\
\text { MET-min }\end{array}$ & $\begin{array}{c}\text { Vigorous } \\
\text { MET-min }\end{array}$ & $\begin{array}{c}\text { Moderate } \\
\text { MET-min }\end{array}$ & $\begin{array}{c}\text { Vigorous } \\
\text { MET-min }\end{array}$ \\
\hline Visceral fat (range) & -0.379 & - & - & -0.339 \\
\hline Fat mass (kg) & -0.355 & - & - & - \\
\hline Fat (\% from body mass) & -0.359 & - & - & - \\
\hline Trunk fat mass (kg) & -0.348 & - & - & - \\
\hline Left arm fat mass (kg) & -0.373 & - & - & - \\
\hline Right arm fat mass (kg) & -0.316 & - & - & - \\
\hline Left leg fat mass (kg) & -0.329 & - & - & - \\
\hline Right leg fat mass (kg) & -0.314 & - & - & - \\
\hline Lean body mass (kg) & - & - & 0.337 & - \\
\hline Trunk muscle mass (kg) & - & - & 0.305 & - \\
\hline Left leg muscle mass (kg) & - & 0.319 & 0.299 & - \\
\hline Right leg muscle mass (kg) & - & 0.333 & 0.348 & - \\
\hline
\end{tabular}

$p<0.05$ for all presented correlation coefficients.

In women, an inverse correlation was noted between visceral fat range and vigorous activity MET minutes/week, whereas a positive correlation was found between lean body, trunk, and leg muscle mass and moderate PA.

\section{Discussion}

A slightly higher proportion of participants in the present study fulfilled the criteria for classification as adequately active (moderate or highly active) than that in the European Union population in general and inhabitants of Latvia in particular [7]. Our study included only participants under age 50 years in good health, which could have led to the recruitment of more active individuals who were more inclined towards and interested in a healthpromoting lifestyle; thus, our PA data may have been improved compared to those in the Eurobarometer 2013 survey. The higher self-reported vigorous PA in men than in women in this study conforms to the Eurobarometer findings [8]. The comparably higher number of minutes of walking and moderate PA in women could be reflective of their lifestyle and exercise preferences; according to the results of another study, their PA level might be strongly influenced by availability of fitness and PA facilities in their immediate living area [9]. A similar number of men and women described their occupation as involving moderate or vigorous PA. However, in the same occupational group, the greater effort in men cannot be ignored. Additionally, moderate to vigorous non-OPA might be more common in men. This could be partially explained by the fact that enjoyment is the motivational factor that stimulates higher-intensity PA in men [10]. Analysis of a single week's data should consider that PA patterns can vary over time (week, seasons, etc.); however, individual activity behaviours of adults could be significantly influenced by occupational rhythms. The more time one spends sitting and less time one spends for walking, moderate, and total PA on working days increases the risk of a decreased weekly PA. There is no clear evidence of a pattern in which a high PA level on working days leads to inactivity during days off 
or vice versa. A large-scale national study in the United States revealed that in the most physically active groups of adults, highly active weekdays were followed by a significant decrease in activity over the weekend, whereas in adults with the lowest total volume of activity per week, activity levels were relatively stable [11]. Other studies reported that full-time employment in a mostly sedentary occupation increased the uncompensated sitting time in other activity domains (leisure, transportation, screen time) of the same individuals $[12,13]$.

A low OPA increases one's risk of a decreased weekly PA; however, it did not correlate with body composition in this group of relatively young and healthy adults. Since weight management depends on both energy expenditure and energy intake, it is possible that an adequate regulation of energy intake may sufficiently maintain normal body weight even if one's PA is low. In the present study, body parameters such as BMI, body lean mass, and fat tissue amount fat mass did not differ among OPA groups. BMI is routinely used to detect underweight or overweight status, but since it is not sensitive to particular tissue type changes, in this study, more specific obesity-related indicators such as body fat percentage and fat amount in different body segments were used to examine the relationship between body composition parameters and PA type.

Durations of moderate and vigorous PA influenced body composition parameters of both sexes. Men who reported more moderate PA had a lower visceral fat rating, body fat mass, fat percentage, and fat in all body segments, whereas vigorous PA correlated with higher leg muscle mass. In women, a higher amount of moderate PA correlated with a higher lean body mass and trunk and leg muscle mass, while vigorous PA inversely correlated with visceral fat range. Correlation differences due to sex can be attributed to reliance on different metabolic substrates for energy production depending on PA duration and intensity as well as sexspecific proportions of fat and muscle tissue and their placement in the body. Women's bodies normally contain more fat tissue, and there is evidence that over the wide range of exercise intensities, lipids remain a dominant source of fuel for oxidative metabolism in women versus men $[14,15]$.

Fat is the main energy source for moderate-intensity PA, in which oxygen uptake is $<65 \%$ of maximum $[16,17]$. Exercise duration determines the ratio of intra- and extramuscular fat input for fat oxidation. For exercises longer than 90-120 minutes, extramuscular fat delivery becomes more important [18], what might lead to decrease in fat mass not only in the exercising extremity but all over the body. Investigations in young non-obese men and women found greater susceptibility of the subcutaneous fat in the legs of men to epinephrine than that in the legs of women; epinephrine induces free fatty acid release in the arm fatty tissue comparably in both sexes. Men also show greater epinephrine release during exercise than women, which resulted in more significant fat breakdown in the legs during exercise for men than in women in the majority of studies using microdialysis or isotope tracers [19-21]. Moreover, free fatty acid release from subcutaneous fat in the upper body was greater in males than in females [22], which could lead to decreased fat mass in the arms of subjects with greater PA.

Meta-analysis of data from a large number of studies revealed that male sex is more receptive to exercise and diet interventions resulting in a larger reduction of total and visceral fat mass [23, 24].

Different types of physical loads have distinct effects on the skeletal muscles. Physical exercises with increased resistance stimulate protein synthesis in the muscle fibres, leading to muscle hypertrophy [25]. High and moderate resistance result in the greatest hypertrophy, while light resistance has a small effect [26]. Exercise duration is also important: If 
high-intensity exercise is performed for a short time or fewer repetitions, the hypertrophic effect is smaller than that of moderate intensity with longer duration or more repetitions.

Aerobic exercise increase can cause muscle hypertrophy comparable to that of resistance training $[27,28]$. Activities that lead to hypertrophy should be intense (70-80\% of the heart rate reserve) maintained for 45 minutes a day for at least 4 days a week. This may explain the greater muscle mass in the legs of men who spend more MET minutes in vigorous PA. In women, the lack of an association between vigorous MET minutes and muscle mass can be explained by reduced quantities of such activities in a typical week. Instead, they reported engaging in great amounts of moderate PA, which could lead to muscle mass increases, and we observed a correlation between moderate PA and higher lean and muscle mass in women.

\section{Conclusions}

The findings of this study indicate that, independent of OPA, men tend to engage in vigorous PA more than women. Adults who are involved in professional duties with insignificant PA have longer sitting time on their working days, less walking and moderate PA, and lower PA overall compared to those with moderate to vigorous OPA. A sex-dependent association was detected between amount of particular PA intensity and muscle or fat tissue distribution. In men, a greater duration of moderate PA was associated with lower amounts of total, visceral, and segmental body fat, whereas women who engaged more in moderate PA had higher lean body mass and segmental muscle masses of the trunk and legs. Greater time spent in vigorous PA was associated with higher leg muscle mass in men and lower visceral fat in women. These observed associations might be used to develop interventions to promote sex- and occupationspecific PA.

\section{References}

[1] S.M. Grundy, Trends Cardiovas. Med. 26(4), 364-373 (2016)

[2] C.A. Slentz, B.D. Duscha, J.L. Johnson, K. Ketchum, L.B. Aiken, G.P. Samsa et al., Arch. Intern. Med. 164(1), 31-39 (2004)

[3] K. Borodulin, T. Laatikainen, A. Juolevi, P. Jousilahti, Eur. J. Public Health 18(3), 339-344 (2008)

[4] C.L. Craig, S.J. Russell, C. Cameron, A. Bauman, Can. J. Public Health; January/February 2004, 95(1) (2004)

[5] S.A. Prince, J.L. Reed, C. McFetridge, M.S. Tremblay, R.D. Reid, Obes. Rev. 18(8), 915-935 (2017)

[6] IPAQ Group, http: //www .ipaq.ki.se (2015)

[7] V. Gerovasili, I.T. Agaku, C.I. Vardavas, F.T. Filippidis, Prev. Med. 81, 87-91 (2015)

[8] European Commission http://ec.europa.eu/commfrontoffice/publicopinion/ archives/ebs/ebs_412_en.pdf (2014)

[9] J.M. Oppert, M.A. Charles, H. Charreire, M. Menai, I. De Bourdeaudhuij, S. Brage, B. de Lauzon-Guillain, G. Fagherazzi, B. Balkau, Int. J. Environ. Res. Public Health 13(8) (2016)

[10] T. Schwaneberg, F. Weymar, S. Ulbricht, M. Dörr, W. Hoffmann, N. van den Berg, Prev. Med. Rep. 7, 162-168 (2017)

[11] K.R. Evenson, F. Wen, J.S. Metzger, A.H. Herring, Int. J. Behav. Nutr. Phys. Act. 12, 20-20 (2015)

[12] M. Saidj, M. Menai, H. Charreire, C. Weber, C. Enaux, M. Aadahl, E. Kesse-Guyot, S. Hercberg, C. Simon J.M. Oppert, BMC Public Health 15, 379 (2015) 
[13] M.P. Jans, K.I. Proper, V.H. Hildebrandt, Am. J. Prev. Med. 33(6), 450-454 (2007)

[14] M.C. Venables, J. Achten, A.E. Jeukendrup, J. Appl. Physiol. (1985) 98(1), 160-167 (2005)

[15] S.G. Dasilva, L. Guidetti, C.F. Buzzachera, H.M. Elsangedy, K. Krinski, W. De Campos, F.L. Goss, C. Baldari, J. Strength Cond. Res. 25(9), 2544-2551 (2011)

[16] J. Achten, M. Gleeson, A.E. Jeukendrup, Med. Sci. Sports Exerc. 34(1), 92-97 (2002)

[17] L.L. Spriet, Sports Med. 44(Suppl 1), 87-96 (2014)

[18] M.J. Watt, G.J.F. Heigenhauser, D.J. Dyck, L.L. Spriet, J. Physiol. 541 (Pt 3), 969-978 (2002)

[19] P. Arner, E. Kriegholm, P. Engfeldt, J. Bolinder, J. Clin. Invest. 85(3), 893-898 (1990)

[20] S.L. Carter, C. Rennie, M.A. Tarnopolsky, Am. J. Physiol. Endocrinol. Metab. 280(6), E898-E907 (2001)

[21] L. Hellstrom, E. Blaak, E. Hagstrom-Toft, Int. J. Sports. Med. 17(6), 439-447 (1996)

[22] E. Blaak, Curr. Opin. Clin. Nutr. Metab. Care 4(6), 499-502 (2001)

[23] R.J. Verheggen, M.F. Maessen, D.J. Green, A.R. Hermus, M.T. Hopman, D.H. Thijssen, Obes. Rev. 17(8), 664-690 (2016)

[24] D. Vissers, W. Hens, J. Taeymans, J.P. Baeyens, J. Poortmans, L. Van Gaal, PLoS One 8(2), e56415 (2013)

[25] H. Ozaki, T. Abe, A.E. Mikesky, A. Sakamoto, S. Machida, H. Naito, J. Phys. Fitness Sports Med. 4(1), 43-51 (2015)

[26] G.E. Campos, T.J. Luecke, H.K. Wendeln, K. Toma, F.C. Hagerman, T.F. Murray, K.E. Ragg, N.A. Ratamess, W.J. Kraemer, R.S. Staron, Eur. J. Appl. Physiol. 88(1), 50-60 (2002)

[27] J.D. Crane, L.G. Macneil, M.A. Tarnopolsky, J. Gerontol. A. Biol. Sci. Med. Sci. 68(6), 631-638 (2013)

[28] A.R. Konopka, M.P. Harber, Exerc. Sport Sci. Rev. 42(2), 53-61 (2014) 